\title{
Genome-wide 5-hydroxymethylcytosine modification pattern is a novel epigenetic feature of globozoospermia
}

\author{
Xiu-Xia Wang ${ }^{1}$, Bao-Fa Sun ${ }^{2}$, Jiao Jiao ${ }^{1}$, Ze-Chen Chong ${ }^{2}$, Yu-Shen Chen ${ }^{2}$, \\ Xiao-Li Wang ${ }^{2}$, Yue Zhao ${ }^{3}$, Yi-Ming Zhou ${ }^{4}$, Da $\mathbf{L i}^{1}$ \\ ${ }^{1}$ Department of Obstetrics and Gynecology, Shengjing Hospital of China Medical University, Shenyang 110004, China \\ ${ }^{2}$ Key Laboratory of Genomic and Precision Medicine, Beijing Institute of Genomics, Chinese Academy of Sciences, Beijing \\ 101300, China \\ ${ }^{3}$ Department of Cell Biology, Key Laboratory of Cell Biology, Ministry of Public Health, and Key Laboratory of Medical Cell \\ Biology, Ministry of Education, China Medical University, Shenyang 110001, China \\ ${ }^{4}$ Department of Medicine, Brigham and Women's Hospital, Harvard Institutes of Medicine, Harvard Medical School, Boston, \\ MA 02115, USA
}

Correspondence to:

Da Li, e-mail: leeda@ymail.com

Keywords: 5-hydroxymethylcytosine, epigenetic, genomic imprinting, globozoospermia

Received: December 23, $2014 \quad$ Accepted: January 17, $2015 \quad$ Published: February 02, 2015

\section{ABSTRACT}

Discovery of 5-hydroxymethylcytosine ( $5 \mathrm{hmC}$ ) in mammalian genomes has excited the field of epigenetics, but information on the genome-wide distribution of $5 \mathrm{hmC}$ is limited. Globozoospermia is a rare but severe cause of male infertility. To date, the epigenetic mechanism, especially $5 \mathrm{hmC}$ profiles involved in globozoospermia progression, remains largely unknown. Here, utilizing the chemical labeling and biotin-enrichment approach followed by Illumina HiSeq sequencing, we showed that (i) 6664, 9029 and 6318 genes contain $5 \mathrm{hmC}$ in normal, abnormal, and globozoospermia sperm, respectively; (ii) some $5 \mathrm{hmC}$-containing genes significantly involves in spermatogenesis, sperm motility and morphology, and gamete generation; (iii) $5 \mathrm{hmC}$ is exclusively localized in sperm intron; (iv) approximately $\mathbf{4 0} \%$ imprinted genes have $5 \mathrm{hmC}$ modification in sperm genomes, but globozoospermia sperm exhibiting a large portion of imprinted genes lose the $5 \mathrm{hmC}$ modification; (v) six imprinted genes showed different $5 \mathrm{hmC}$ patterns in abnormal sperm (GDAP1L1, GNAS, KCNK9, LIN28B, RB1, RTL1), and five imprinted genes showed different $5 \mathrm{hmC}$ patterns in globozoospermia sperm (KCNK9, LIN28B, RB1, SLC22A18, ZDBF2). These results suggested that differences in genome-wide $5 \mathrm{hmC}$ patterns may in part be responsible for the sperm phenotype. All of this may improve our understanding of the basic molecular mechanism underlying sperm biology and the etiology of male infertility.

\section{INTRODUCTION}

Globozoospermia (also called round-headed sperm syndrome), characterized by $100 \%$ round-headed spermatozoa and lack of acrosome, is a rare but severe cause of male infertility [1]. Familial globozoospermia a suggests that globozoospermia most probably originates in spermatogenesis, specifically in acrosome formation and sperm head elongation, which is largely determined by genetic and epigenetic factors [1-4]. As is already known, sperm carry distinctive epigenetic modifications that are adjusted by reprogramming during the spermatogenesis and fertilization process [1,2]. However, to date, little is known about epigenomics, especially the 5-hydroxymethylcytosine $(5 \mathrm{hmC})$ profiles in the pathophysiology of globozoospermia. $5 \mathrm{hmC}$, a novel modified cytosine, is oxidized from 5-methylcytosine by the ten-eleven translocation family of proteins, and the discovery of $5 \mathrm{hmC}$ in mammalian genomes has excited the field of epigenetics [5]. In addition, $5 \mathrm{hmC}$, as a unique and dynamic mark of cellular state, has been shown to be involved in diverse cellular processes, including transcriptional regulation, DNA methylation regulation, stem cell pluripotency and tumorigenesis [6]. Notably, recent studies have suggested that highly ordered alterations of $5 \mathrm{hmC}$ are potentially responsible 
for the differentiation of spermatogenic cells [7]. For this reason, the present study was undertaken to investigate comprehensive $5 \mathrm{hmC}$ profiling in normal, abnormal, and globozoospermia sperm by a chemical-labeling and biotin-enrichment approach followed by Illumina HiSeq sequencing, and to provide novel insight into the epigenetic-mediated dysfunction in the pathogenesis of globozoospermia.

\section{RESULTS}

\section{Isolation and identification of normal, abnormal, and globozoospermia sperm}

All normal sperm clearly have an oval head with a long tail (Figure 1A). While various misshapen head or tail defects such as amorphous head, crooked and double tail were observed in abnormal sperm (Figure 1B). The globozoospermia sperm had round heads (Figure 1C). In all, these three sets of sperm represent typical sperm types with different fertility ability and were used for studying the impact of $5 \mathrm{hmC}$ on male infertility.

\section{$5 \mathrm{hmC}$ enrichment and sequencing in normal, abnormal, and globozoospermia sperm}

To assess the general content of $5 \mathrm{hmC}$, we first evaluated the existence of $5 \mathrm{hmC}$ in sperm genome by dot blot assay (Figure 2A). We detected a significant quantity of $5 \mathrm{hmC}$ in as little as $100 \mathrm{ng}$ sperm genomic DNA. It is quite interesting to note that the amount of $5 \mathrm{hmC}$ is remarkably changed in different tissues in contrast to the stable patterns of 5-methylcytosine $(5 \mathrm{mC})$ [8].

To generate genome-wide maps of $5 \mathrm{hmC}$ in sperm genome, we used a well-established chemical-labeling and biotin-enrichment approach to enrich $5 \mathrm{hmC}$ containing DNA fragments from normal, abnormal, and globozoospermia genomic DNA and subjected them to high throughput sequencing. Generally, we got 45 million to 60 million sequencing reads and mapped these to human genome with approximately $90 \%$ successful mapping rates (Figure 2B). We identified $5 \mathrm{hmC}$ enriched peaks using a model-based analysis of CHIP-seq software (MACS) $\left(P<10^{-5}\right.$, fold enrichment $\left.>10\right)$. In total, we identified 20486, 38282 and 19354 peaks in normal, abnormal, and globozoospermia sperm, respectively (Figures 2C and 2D, Supplementary Table S1).

\section{Genomic features of $\mathbf{5 h m C}$ in normal, abnormal, and globozoospermia sperm}

We plotted those $5 \mathrm{hmC}$ peaks on Ref Seq annotated genes and identified 6664, 9029 and 6318 genes containing $5 \mathrm{hmC}$ in normal, abnormal, and globozoospermia sperm, respectively (Figure $3 \mathrm{~A}$ ), of which there was an especially strong overlap with 3576 genes in all these $5 \mathrm{hmC}$ gene pools (Figure $3 \mathrm{~B}$ ). The total and specific $5 \mathrm{hmC}$-containing gene lists are shown in Supplementary Table S2. Furthermore, analysis of genome-wide $5 \mathrm{hmC}$-containing genes shows that $5 \mathrm{hmC}$ are not distributed randomly on chromosomes, but exhibit a unique pattern on specific chromosomes (Figure 3C). With regards to the distribution region, it is striking that most of $5 \mathrm{hmC}$ peaks are located in introns (Figure 3D), whereas in ES cells $5 \mathrm{hmC}$ is preferentially present in the upstream of gene bodies and in the brain it is enriched in gene bodies $[9,10]$.

\section{GO analysis of $5 \mathrm{hmC}$-containing genes in normal, abnormal, and globozoospermia sperm}

The preferential distribution of $5 \mathrm{hmC}$ in introns in sperm genome suggested it may have distinct roles in sperm maturation and function. As shown in Figure 4A and Supplementary Table S3, 5hmC-containing genes in all three genomes share cell motion and signal transduction pathways, indicating $5 \mathrm{hmC}$ has conserved
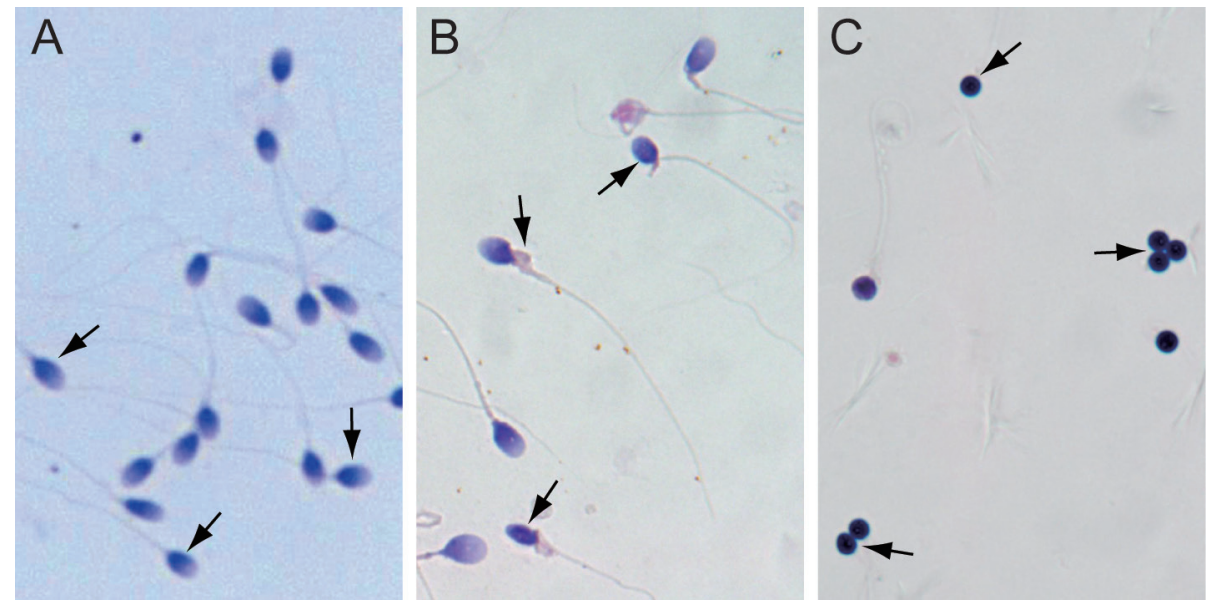

Figure 1: Isolation of normal sperm, abnormal sperm, and globozoospermia sperm. (A) Normal sperm. (B) Abnormal sperm. (C) Globozoospermia sperm. 


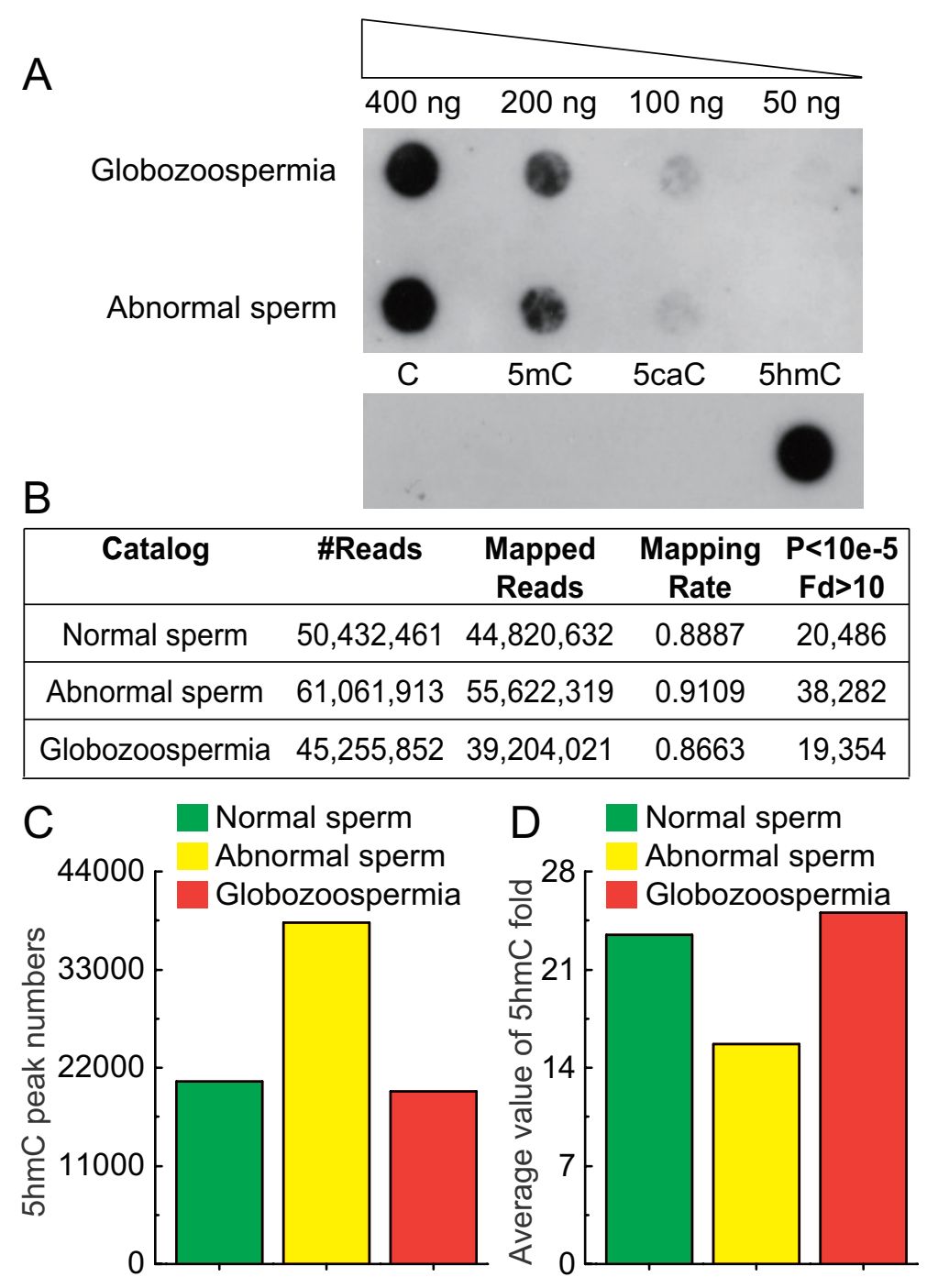

Figure 2: Sequencing results of $5 \mathrm{hmC}$ in normal, abnormal, and globozoospermia sperm genomes. (A) Dot blot detection of $5 \mathrm{hmC}$ in sperm DNA with positive and negative control. $400 \mathrm{ng}, 200 \mathrm{ng}, 100 \mathrm{ng}$ and $50 \mathrm{ng}$ genomic DNA purified from abnormal and globozoospermia sperm were blotted on Hybond-N+ nylon-based membrane and immune-blotted using anti-5-hydroxymethylytosine antibody. Synthesized oligonucleotides with and without $5 \mathrm{hmC}$ modification were included as positive and negative controls. (B) Mapping results of $5 \mathrm{hmC}$ sequencing in normal, abnormal, and globozoospermia sperm genomes. Raw reads were aligned to human UCSC hg 19 and peaks calling using MACS $\left(P<10^{-5}\right.$, fold enrichment $\left.>10\right)$. (C) $5 \mathrm{hmC}$ peak numbers in normal, abnormal, and globozoospermia sperms. 20486, 38282 and 19354 peaks were identified in normal, abnormal, and globozoospermia sperm, respectively. (D) Average value of $5 \mathrm{hmC}$ fold enrichment in normal, abnormal, and globozoospermia sperm.

but important roles in sperm motion and communication. Notably, cellular component organization is lost in globozoospermia but present in normal and abnormal sperm, whereas cell adhesion and response to (chemical) stimulus pathways is additionally involved in abnormal and globozoospermia without normal sperm.

To evaluate aberrant $5 \mathrm{hmC}$ modification in sperm dysregulation, we further performed GO analysis of specific 5hmC-containing genes in normal, abnormal, and globozoospermia sperm genome (Figure 4B, Supplementary Table S3). We found the organic substance metabolic process pathway is most significantly anomalous in normal, abnormal, and globozoospermia sperm. In particular, 10 gamete generation genes are implicated in abnormal sperm (Table 1), suggesting aberrant $5 \mathrm{hmC}$ modification of these genes may affect gamete generation, potentially leading to sterility of abnormal sperms.

\section{5hmC-containing genes overlap with imprinted genes among normal, abnormal, and globozoospermia sperm}

To evaluate $5 \mathrm{hmC}$ modification alteration in imprinted genes, we compared $5 \mathrm{hmC}$-containing genes with 96 known imprint genes from the imprinted gene database (http://www.geneimprint.com/site/home), and visualized by area-proportional Venn diagrams using an online tool BioVenn. In total, approximately 


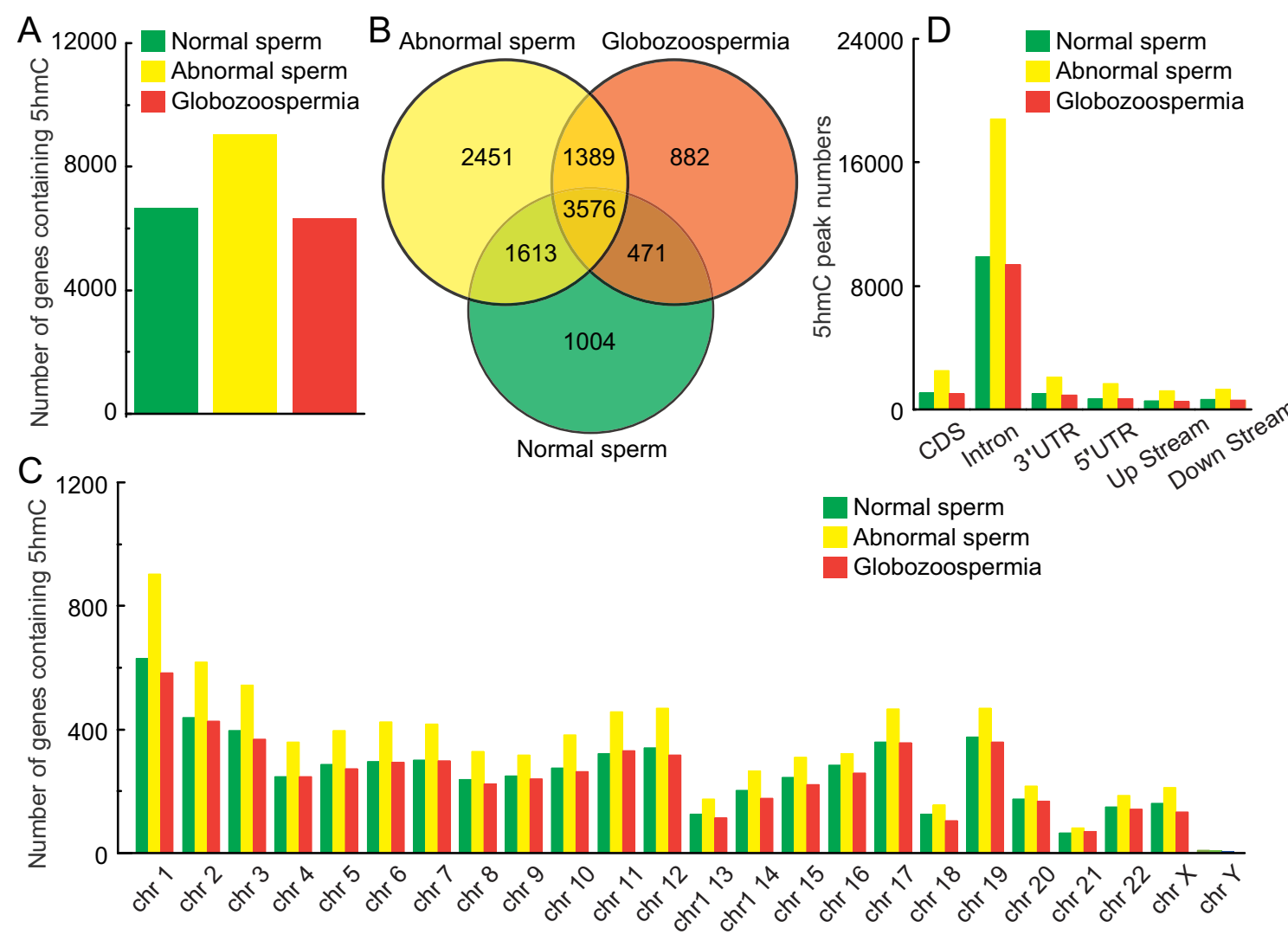

Figure 3: Genomic features of $5 \mathrm{hmC}$ in normal, abnormal, and globozoospermia sperm genomes. (A) Number of genes containing $5 \mathrm{hmC}$ in normal, abnormal, and globozoospermia sperm genomes. (B) Overlap comparison of 5hmC-containing imprinted genes in normal, abnormal, and globozoospermia sperm genomes. (C) Number of genes containing $5 \mathrm{hmC}$ in each chromosome in normal, abnormal, and globozoospermia sperm genomes. (D) 5hmC peak numbers in CDS, intron, 3'UTR, 5'UTR, upstream (200 bp) and downstream (200 bp) in normal, abnormal, and globozoospermia sperm genomes.

$40 \%$ of the imprinted genes (38 imprinted genes) are $5 \mathrm{hmC}$-containing genes in normal, abnormal, and globozoospermia sperm genomes (Figure 5A). In detail, 30,30 and 21 imprinted genes contained $5 \mathrm{hmC}$ in normal, abnormal, and globozoospermia sperm genomes, respectively (Table 2). The Venn diagram shows that normal, abnormal, and globozoospermia sperm share 14 imprinted genes (Figure 5A). Compared with normal sperm, 6 imprinted genes lost $5 \mathrm{hmC}$ modification, while another 6 imprinted genes gained $5 \mathrm{hmC}$ modification in abnormal sperm (Figure 5B). Interestingly, compared with normal sperm, a large portion (14 out of 30) of imprinted genes lost $5 \mathrm{hmC}$ modification and 5 imprinted genes gained $5 \mathrm{hmC}$ modification in globozoospermia patient (Figure 5C), suggesting that the loss of $5 \mathrm{hmC}$ in imprinted genes may be associated with globozoospermia.

\section{DISCUSSION}

Emerging evidence indicates that epigenetic mechanisms, especially the aberrant DNA methylation $(5 \mathrm{mC})$ of imprinted genes in sperm DNA, play an important part in abnormal sperm parameters and male infertility [11]. However, it is interesting to note that sperm methylation profiles have been recently described [12], but to date, no studies have examined the distribution and features of $5 \mathrm{hmC}$ in sperm genome, and few studies have linked $5 \mathrm{hmC}$ to male infertility. The current study generated the first landscape of $5 \mathrm{hmC}$ in normal, abnormal, and globozoospermia sperm genomes, and provided novel insights into $5 \mathrm{hmC}$-related sperm physiology and pathology.

In this study, we identified 6664, 9029 and 6318 genes containing $5 \mathrm{hmC}$ in normal, abnormal, and globozoospermia sperm, respectively. Notably, $5 \mathrm{hmC}$ is exclusively localized in intron, a distinct characteristic difference from previous reports that $5 \mathrm{hmC}$ is preferentially enriched within exons and near transcriptional start sites in embryonic stem cells [10], and alteration of $5 \mathrm{hmC}$ modification occurred mainly at gene bodies, along with environmental changes [13] and neurons development [14]. In addition, the discrete distribution of $5 \mathrm{hmC}$ in the intron regions of sperm genome suggested that it may have specific roles in sperm maturation and function.

We also found that $5 \mathrm{hmC}$-containing genes are involved in various functional pathways, some of which have important roles in sperm, such as spermatogenesis, 
A○ Normal sperm

Phosphate metabolic process (206)

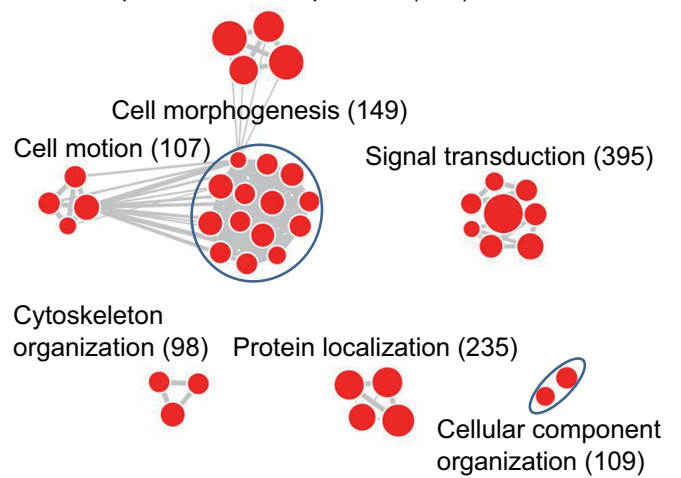

\section{A○Abnormal sperm}

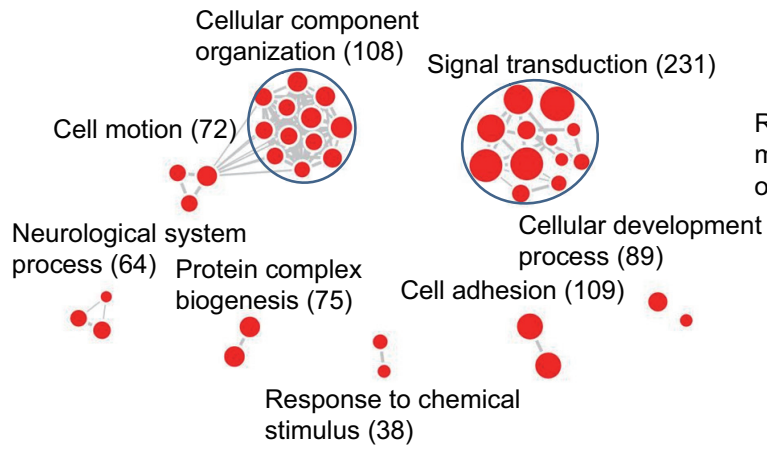

\section{A๑ Globozoospermia}

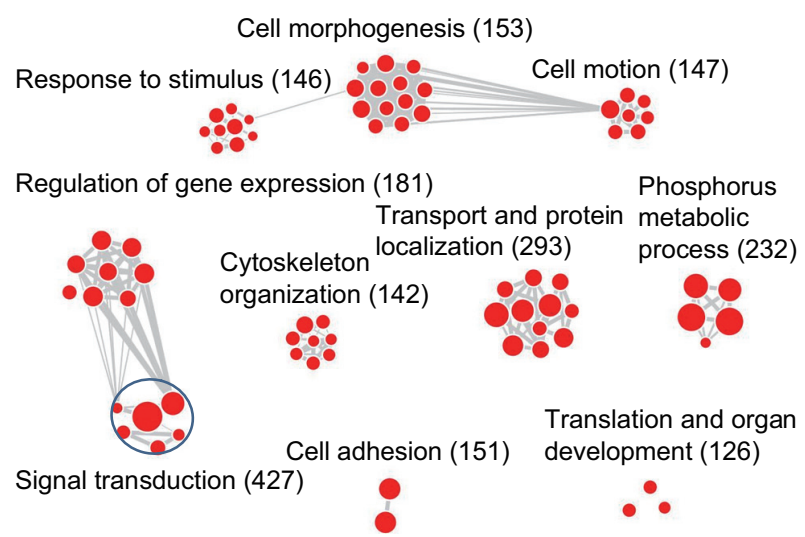

B Normal sperm

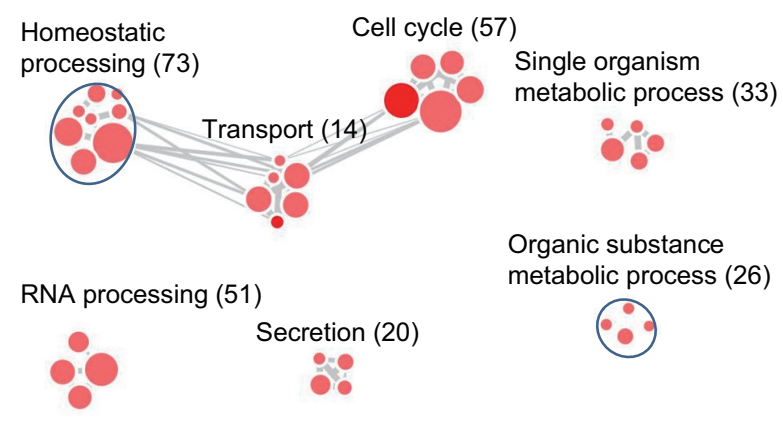

B Abnormal sperm

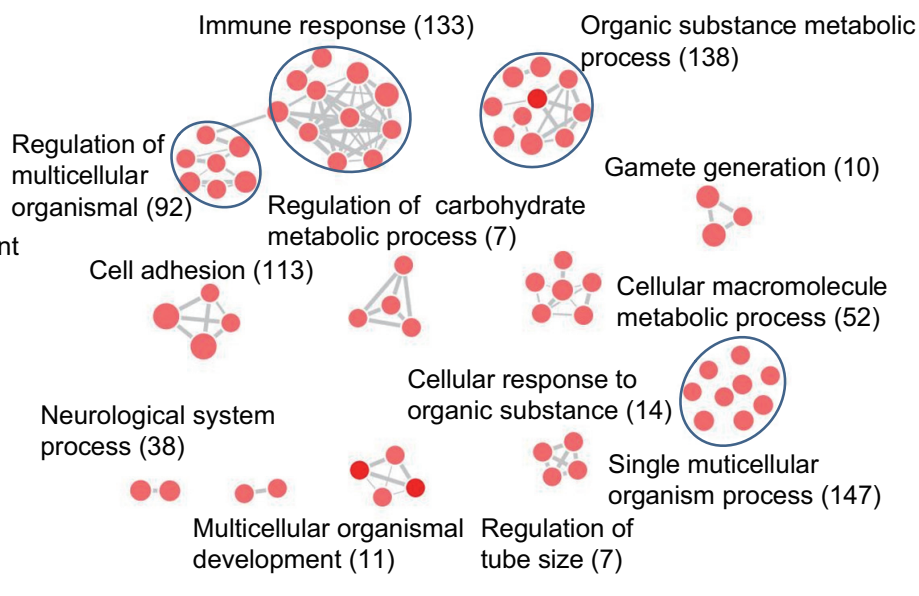

$B \odot$ Globozoospermia

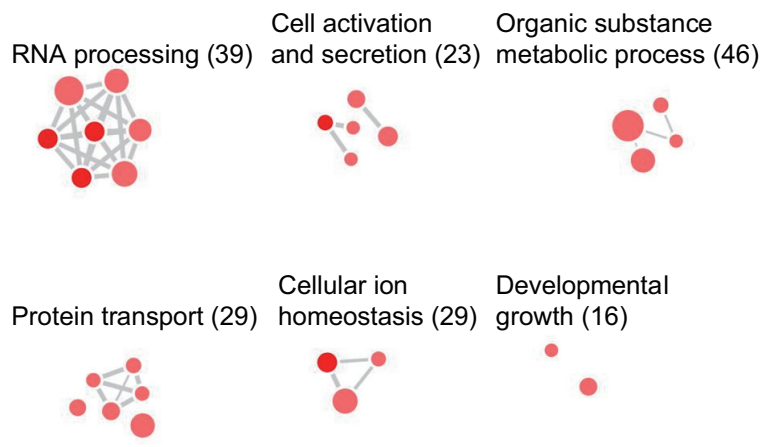

Figure 4: GO analysis of total and specific 5hmC-containing genes in normal, abnormal, and globozoospermia sperm genomes. (A) $\mathrm{GO}$ analysis of total $5 \mathrm{hmC}$-containing genes in normal, abnormal, and globozoospermia sperm genomes. (B) GO analysis of specific $5 \mathrm{hmC}$-containing genes in normal, abnormal, and globozoospermia sperm genomes.

sperm motility and morphology, and sperm cell maturation. For instance, (i) growth differentiation factor 9 (GDF9) is significantly associated with sperm quality traits, and is involved in the initiation or maintenance of spermatogenesis [15]; (ii) methylation patterns of the nuclear ribonucleoprotein polypeptide N (SNRPN) promoters are associated with changes in sperm motility and morphology, which could lead to male infertility [16]; (iii) membrane-associated guanylate kinase, WW and PDZ domain containing 2 (MAGI-2), which is known to localize at the tight junction of epithelial cells, plays an important part in sperm cell maturation [17]; (iv) FIGLA (factor in the germ line, alpha) encodes a germ cellspecific basic helix-loop-helix transcription factor, which has essential roles in the repression of sperm-associated genes during normal postnatal oogenesis [18]. 
Table 1: List of $5 \mathrm{hmC}$ containing genes associated with gamete generation in abnormal sperm

\begin{tabular}{l|l|}
\hline Gene symbol & Description \\
\hline NOBOX & $\begin{array}{l}\text { Homeobox protein NOBOX, Homeodomain-containing protein OG-2, Newborn ovary homeobox } \\
\text { protein, Oocyte-specific homeobox protein }\end{array}$ \\
\hline HEXB & $\begin{array}{l}\text { Beta-hexosaminidase subunit beta, Beta-N-acetylhexosaminidase subunit beta, Hexosaminidase subunit } \\
\text { B, Cervical cancer proto-oncogene 7 protein, N-acetyl-beta-glucosaminidase subunit beta }\end{array}$ \\
\hline GDF9 & Growth/differentiation factor 9 (GDF-9) \\
\hline REC8 & Meiotic recombination protein REC8 homolog (Cohesin Rec8p) \\
\hline SOHLH2 & Spermatogenesis- and oogenesis-specific basic helix-loop-helix-containing protein 2 \\
\hline FIGLA & $\begin{array}{l}\text { Factor in the germline alpha, Class C basic helix-loop-helix protein 8, Folliculogenesis-specific basic } \\
\text { helix-loop-helix protein, Transcription factor FIGa }\end{array}$ \\
\hline ANG & Angiopoietin-4 (ANG-4) (Angiopoietin-3) (ANG-3) \\
\hline ZGLP1 & GATA-type zinc finger protein 1 (GATA-like protein 1) (GLP-1) \\
\hline PAQR7 & $\begin{array}{l}\text { Membrane progestin receptor alpha (mPR alpha) (Progestin and adipoQ receptor family member 7) } \\
\text { (Progestin and adipoQ receptor family member VII) }\end{array}$ \\
\hline EREG & Proepiregulin \\
\hline
\end{tabular}
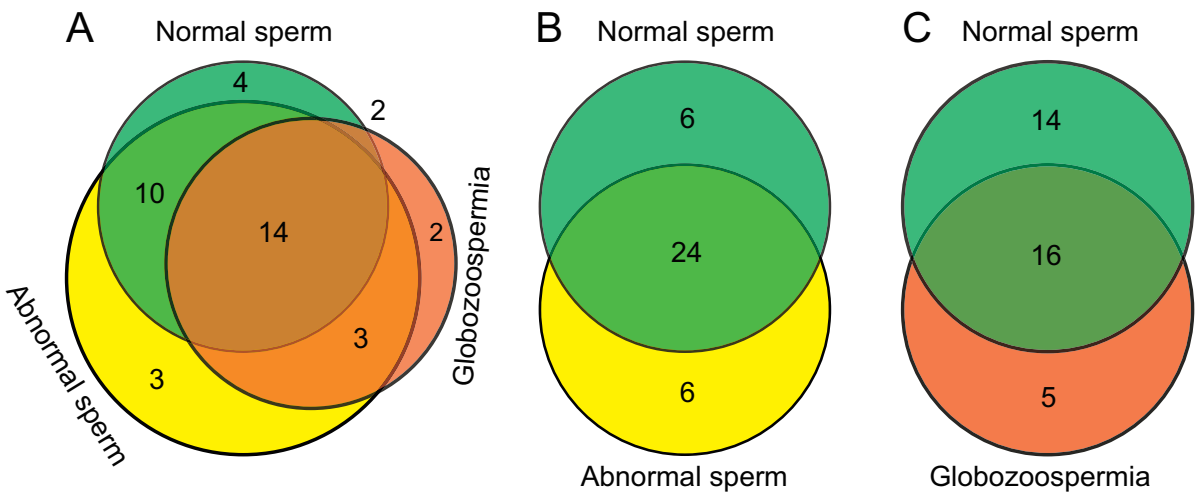

Figure 5: Overlap comparison of 5hmC-containing imprinted genes among normal, abnormal, and globozoospermia sperm by Venn diagrams. (A) Overall comparison of $5 \mathrm{hmC}$-containing imprinted genes among normal, abnormal, and globozoospermia sperm. (B) Overlap comparison of 5hmC-containing imprinted genes between normal and abnormal sperm. (C) Overlap comparison of $5 \mathrm{hmC}$-containing imprinted genes between normal and globozoospermia sperm.

It is well known that aberrant DNA methylation patterns, mainly in imprinted genes, have been associated with sperm dysfunction. Therefore, we further compared the 5hmC-containing genes among normal, abnormal, and globozoospermia sperm genomes, referring to the imprinted gene database. We found that approximately $40 \%$ (38 out of 96) imprinted genes had $5 \mathrm{hmC}$ modification in normal, abnormal, and globozoospermia sperm genomes. The globozoospermia sperm showed that a large portion (14 out of 30) of imprinted genes lose $5 \mathrm{hmC}$ modification, compared with normal sperm, suggesting that the loss of $5 \mathrm{hmC}$ in imprinted genes may have essential roles in globozoospermia progression. As shown in Table 2 , six imprinted genes showed different $5 \mathrm{hmC}$ patterns between normal and abnormal sperm (GDAP1L1, GNAS, KCNK9, LIN28B, RB1, RTL1), and five imprinted genes showed different $5 \mathrm{hmC}$ patterns between normal and globozoospermia sperm (KCNK9, LIN28B, RB1, SLC22A18, ZDBF2). These data may help to identify several novel epigenetically regulated genes that are possibly involved in abnormal sperm and globozoospermia sperm, and these aberrant $5 \mathrm{hmC}$-related genes may also be potential biomarkers of abnormal sperm parameters.

Taken together, our study provided a genomewide distribution of $5 \mathrm{hmC}$ in normal, abnormal, and globozoospermia sperm, and understanding the epigenetic mechanisms may yield new insight into the sperm biology and etiology of male infertility. 
Table 2: List of imprinted genes overlapped with $5 \mathrm{hmC}$ containing genes in sperm

\begin{tabular}{|c|c|c|}
\hline Normal sperm & Abnormal sperm & Globozoospermia \\
\hline AIM1 & AIM1 & ANO1 \\
\hline ANO1 & ANO1 & ATP10A \\
\hline ATP10A & ATP10A & DDC \\
\hline BLCAP & CDKN1C & DLGAP2 \\
\hline CDKN1C & DDC & GLIS3 \\
\hline DDC & DLGAP2 & IGF2R \\
\hline DGCR6L & GDAP1L1 & INPP5F \\
\hline DLGAP2 & GLIS3 & KCNK9 \\
\hline DNMT1 & GNAS & KCNQ1 \\
\hline GLIS3 & GPR1 & LIN28B \\
\hline GPR1 & GRB10 & MAGI2 \\
\hline GRB10 & IGF2R & NLRP2 \\
\hline IGF2R & KCNK9 & NTM \\
\hline INPP5F & KCNQ1 & OSBPL5 \\
\hline KCNQ1 & LIN28B & PPP1R9A \\
\hline MAGI2 & MAGI2 & RB1 \\
\hline MEST & MEST & SLC22A18 \\
\hline NLRP2 & NLRP2 & SLC22A3 \\
\hline NTM & NTM & TP73 \\
\hline OSBPL5 & OSBPL5 & ZDBF2 \\
\hline PLAGL1 & PLAGL1 & ZFAT \\
\hline PPP1R9A & PPP1R9A & \\
\hline SLC22A3 & RB1 & \\
\hline SNRPN & RTL1 & \\
\hline TP73 & SLC22A3 & \\
\hline UBE3A & SNRPN & \\
\hline $\mathrm{ZC} 3 \mathrm{H} 12 \mathrm{C}$ & UBE3A & \\
\hline ZFAT & $\mathrm{ZC} 3 \mathrm{H} 12 \mathrm{C}$ & \\
\hline ZIM2 & ZFAT & \\
\hline ZNF597 & ZIM2 & \\
\hline
\end{tabular}

\section{METHODS}

\section{Ethical statements}

The investigation was conducted in accordance with the ethical standards and according to the Helsinki Declaration of 1975, and was approved by the Institutional Review Board at China Medical University.
Preparation of genomic DNA, $5 \mathrm{hmC}$ specific chemical labeling and affinity purification

Genomic DNA was prepared using a Wizard Genomic DNA Purification kit (Promega Cat.:A1120) by following the manufacturer's instructions. Equal amounts of genomic DNA were extracted from $1 \times 10^{5}$ normal, abnormal, and globozoospermia sperm, respectively. 
Purified genomic DNA was sonicated into short fragments by Covaris DNA shearing with microTUBEs according to the manufacturer's instructions. Then $5 \mathrm{hmC}$ labeling reactions were performed in $75 \mu \mathrm{l}$ solution containing $50 \mathrm{~mm}$ HEPES buffer ( $\mathrm{pH} 7.9$ ), $250 \mathrm{~mm} \mathrm{MgCl}, 100 \mu \mathrm{m}$ UDP-6- $\mathrm{N}_{3}$-glucose and $80 \mathrm{U} \beta$-glucosyltransferase. The reaction was incubated for $2 \mathrm{~h}$ at $37^{\circ} \mathrm{C}$. After the reaction, DNA substrates were purified and buffer-exchanged in $\mathrm{H}_{2} \mathrm{O}$ via Bio-Rad-Spin columns according to the manufacturer's instructions. Click chemistry was performed with the addition of $150 \mu \mathrm{m}$ biotin into the DNA solution and incubated for $2 \mathrm{~h}$ at $37^{\circ} \mathrm{C}$. The DNA samples were then purified by Invitrogen Dynabeads MyOne ${ }^{\mathrm{TM}}$ Streptavidin $\mathrm{C} 1$ according to the manufacturer's instructions.

\section{Sequencing of $5 \mathrm{hmC}$-enriched genomic DNA}

5 hmC-enriched genomic DNA libraries were generated following the Illumina protocol for 'Preparing Samples for CHIP Sequencing of DNA'. Then, $20 \mathrm{ng}$ of $5 \mathrm{hmC}$-enriched DNA was used to initiate the protocol. DNA fragments were gel purified after the adapter ligation step. PCR-amplified DNA libraries were quantified on an Agilent 2100 Bio analyzer using a quantitative PCR. We performed $100 \mathrm{bp}$ single end sequencing on Illumina Hiseq2000 to get a 5hmC-enriched DNA fragment sequence.

\section{5hmC reads mapping and peaks calling}

The deep sequencing reads were stripped of the adaptor sequences with FASTX tool kit (http://hannonlab. cshl.edu/fastx_toolkit/). Reads that were less than $25 \mathrm{nt}$ in length or contained an ambiguous nucleotide were discarded. The remaining reads were aligned to human UCSC hg19 genome, with up to two mismatches allowed, by the BWA software [19]. All non-redundant uniquely mapped reads were used for peaks calling using MACS $\left(P<10^{-5}\right)[20]$. Association of $5 \mathrm{hmC}$ peaks with genomic features was performed by overlapping peak locations with known genomic features obtained from hg19 database. Location information of CDS, intron, 3'UTR, 5'UTR, upstream (200 bp), and downstream (200 bp) were downloaded from UCSC.

\section{Immuno-dot-blot assay}

Genomic DNA was denatured in TE buffer for 10 min at $95^{\circ} \mathrm{C}$ and immediately chilled on ice for $5 \mathrm{~min}$. Dot blot was performed on a Bio-Dot Apparatus (\#1706545, Bio-Rad); 50, 100, 200 and $400 \mathrm{ng}$ of each DNA sample was spotted on the positively charged nylon membrane, respectively, then the membrane was baked for $2 \mathrm{~h}$ at $80^{\circ} \mathrm{C}$ until completely dry, followed by UV254 crosslink for 10 min to fix DNA on the membrane. The membrane was then blocked briefly with 5\% non-fat milk for $1.5 \mathrm{~h}$ at room temperature. The primary rabbit anti5-hydroxymethylytosine antibody (1:10000, \#39769, Active Motif) was applied to the membrane and incubated at RT for $1 \mathrm{~h}$ or overnight at $4^{\circ} \mathrm{C}$. After incubation with a peroxidase-conjugated anti-rabbit IgG secondary antibody, the signal was visualized by using ECL (Millipore). The dot-blot densities were analyzed with Image J software. The 5hmC-containing DNA was used as a positive control, and the normal $\mathrm{C}, 5 \mathrm{mC}$, 5-carC-containing DNA were used as the negative controls to verify the specificity of $5 \mathrm{hmC}$ antibody.

\section{Accession number}

All original data sets have been deposited in the Gene Expression Omnibus Database under the accession number GSE46135.

\section{Gene ontology analysis}

Gene ontology analyses were performed on sets of unique RefSeq identifiers using DAVID bioinformatics resources 6.7 functional annotation tools [21]. GO Biological processes and Interpro database were used. Categories with $P<0.05$ were considered statistically significant. The analysis results are visualized as an enrichment map by using Cytoscape software [22].

\section{ACKNOWLEDGMENTS}

This work was supported by the 973 Program of China (No. 2013CB945201), Natural Science Foundation of China (No. 31171259, No. 31271364 and No. 81402130), and Doctoral Startup Foundation of Liaoning Province (No. 20141045).

\section{Conflict of interest}

The authors declare that they have no competing interests.

\section{REFERENCES}

1. Dam AH, Feenstra I, Westphal JR, Ramos L, van Golde RJ, Kremer JA. Globozoospermia revisited. Hum Reprod Update. 2007; 13:63-75.

2. Gu TP, Guo F, Yang H, Wu HP, Xu GF, Liu W, Xie ZG, Shi L, He X, Jin SG, Iqbal K, Shi YG, Deng Z, et al. The role of Tet3 DNA dioxygenase in epigenetic reprogramming by oocytes. Nature. 2011; 477:606-610.

3. Moradan S, Yousefi B. Globozoospermia syndrome: two case reports. J Med Liban. 2014; 62:183-185.

4. Pirrello O, Machev N, Schimdt F, Terriou P, Menezo Y, Viville S. Search for mutations involved in human globozoospermia. Hum Reprod. 2005; 20:1314-1318. 
5. Nakamura T, Liu YJ, Nakashima H, Umehara H, Inoue K, Matoba S, Tachibana M, Ogura A, Shinkai Y, Nakano T. PGC7 binds histone $\mathrm{H} 3 \mathrm{~K} 9 \mathrm{me} 2$ to protect against conversion of $5 \mathrm{mC}$ to $5 \mathrm{hmC}$ in early embryos. Nature. $2012 ; 486: 415-419$.

6. Ye C, Li L. 5-hydroxymethylcytosine: a new insight into epigenetics in cancer. Cancer Biol Ther. 2014; 15:10-5.

7. Gan H, Wen L, Liao S, Lin X, Ma T, Liu J, Song CX, Wang M, He C, Han C, Tang F. Dynamics of 5-hydroxymethylcytosine during mouse spermatogenesis. Nat Commun. 2013; 4:1995.

8. Globisch D, Münzel M, Müller M, Michalakis S, Wagner M, Koch S, Brückl T, Biel M, Carell T. Tissue distribution of 5-hydroxymethylcytosine and search for active demethylation intermediates. PLoS One. 2010; 5:e15367.

9. Booth MJ, Branco MR, Ficz G, Oxley D, Krueger F, Reik W, Balasubramanian S. Quantitative sequencing of 5-methylcytosine and 5-hydroxymethylcytosine at singlebase resolution. Science. 2012; 336:934-937.

10. Pastor WA, Pape UJ, Huang Y, Henderson HR, Lister R, Ko M, McLoughlin EM, Brudno Y, Mahapatra S, Kapranov P, Tahiliani M, Daley GQ, Liu XS, et al. Genome-wide mapping of 5-hydroxymethylcytosine in embryonic stem cells. Nature. 2011; 473:394-397.

11. Laurentino S, Beygo J, Nordhoff V, Kliesch S, Wistuba J, Borgmann J, Buiting K, Horsthemke B, Gromoll J. Epigenetic germline mosaicism in infertile men. Hum Mol Genet. 2014; in press.

12. Molaro A, Hodges E, Fang F, Song Q, McCombie WR, Hannon GJ, Smith AD. Sperm methylation profiles reveal features of epigenetic inheritance and evolution in primates. Cell. 2011; 146:1029-1041.

13. Irier $H$, Street RC, Dave R, Lin L, Cai C, Davis $T H$, Yao B, Cheng Y, Jin P. Environmental enrichment modulates 5-hydroxymethylcytosine dynamics in hippocampus. Genomics. 2014; 104:376-382.
14. Colquitt BM, Allen WE, Barnea G, Lomvardas S. Alteration of genic 5-hydroxymethylcytosine patterning in olfactory neurons correlates with changes in gene expression and cell identity. Proc Natl Acad Sci U S A. 2013; 110:14682-14687.

15. Tang KQ, Yang WC, Zhang XX, Yang LG. Effects of polymorphisms in the bovine growth differentiation factor 9 gene on sperm quality in Holstein bulls. Genet Mol Res. 2013; 12:2189-2195.

16. Botezatu A, Socolov R, Socolov D, Iancu IV, Anton G. Methylation pattern of methylene tetrahydrofolate reductase and small nuclear ribonucleoprotein polypeptide $\mathrm{N}$ promoters in oligoasthenospermia: a case-control study. Reprod Biomed Online. 2014; 28:225-231.

17. Ihara K, Nishimura T, Fukuda T, Ookura T, Nishimori K. Generation of venus reporter knock-in mice revealed MAGI-2 expression patterns in adult mice. Gene Expr Patterns. 2012; 12:95-101.

18. $\mathrm{Hu} \mathrm{W}$, Gauthier L, Baibakov B, Jimenez-Movilla M, Dean J. FIGLA, a basic helix-loop-helix transcription factor, balances sexually dimorphic gene expression in postnatal oocytes. Mol Cell Biol. 2010; 30:3661-3671.

19. Li H, Durbin R. Fast and accurate short read alignment with Burrows-Wheeler transform. Bioinformatics. 2009; 25:1754-1760.

20. Zhang Y, Liu T, Meyer CA, Eeckhoute J, Johnson DS, Bernstein BE, Nusbaum C, Myers RM, Brown M, Li W, Liu XS. Model-based analysis of ChIP-Seq (MACS). Genome Biol. 2008; 9:R137.

21. Huang da W, Sherman BT, Lempicki RA. Systematic and integrative analysis of large gene lists using DAVID bioinformatics resources. Nat Protoc. 2009; 4:44-57.

22. Smoot ME, Ono K, Ruscheinski J, Wang PL, Ideker T. Cytoscape 2, new features for data integration and network visualization. Bioinformatics. 2011; 27:431-432. 